\title{
Isolation and Identification of Antibacterial Compounds Isolated from Endophytic Fungus Emericella qaudrilineata(RS-5)
}

\author{
Jyoti Goutam ${ }^{* 1}$, R N Kharwar ${ }^{1}$, Vinod Kumar Tiwari ${ }^{2}$, Amrita Mishra $^{2}$ and Shweta Singh ${ }^{1}$
}

${ }^{1}$ Laboratory of Mycopathology and Microbial Technology, Center of Advance Study in Botany, Banaras Hindu University, Varanasi, India

${ }^{2}$ Department of chemistry, Banaras Hindu University, Varanasi, India

\begin{abstract}
Present work is directed toward the isolation and identification of biological active (antimicrobial) compound from metabolite extracted from endophytic fungus Emericella qaudrilineata(RS-5) (Accession number KC662361). Crude extracted from endophytic fungus Emericella qaudrilineata possessing antibacterial activity was subjected to bioassay guided fractionation in which toluene fraction exhibited maximum antibacterial activity against Staphylococcus aureus (gram positive) and Aeromonas hydrophilla (gram negative) respectively. Sequentially compounds fractionated from ethyl acetate and hexane also shown feeble antibacterial activity. This reveals that toluene fraction contain active principle of antimicrobial interest. In continuation to above experiment, major active fractions were analyzed with the help of gas chromatography mass spectroscopy (GCMS). GCMS analysis of toluene fraction showed benzyl benzoate $(27.3 \%)$, benzaldehyde dimethyl acetal $(15.04 \%)$ and benzoic acid $(17 \%)$, as the dominant compound present in fraction of toluene. In context to above details separation of compound was performed by silica gel column chromatography. Separated band showed its presence at $0.6 \mathrm{RF}$ value in silica gel (TLC) plates. Separated compound also exhibited remarkable antibacterial activity. The optimized flow rate of column was $20 \mathrm{ml} / \mathrm{min}$ in ratio of hexane 20:80 toulene mobile phase. Prediction of $\mathrm{C}^{13} \mathrm{NMR}, \mathrm{H}^{1} \mathrm{NMR}$ and FTIR data supported the dominating compound of GCMS profile of toulene fraction, and identified it as Benzyl benzoate. Medically benzyl benzoate is an active ingredient of Ascabiol that is generally used to treat scabies various skin diseases.
\end{abstract}

Keywords: Emericella qaudrilineata; Chromatography; Antimicrobial compounds; Benzyl benzoate

\section{Introduction}

"Endophytes" are the microorganism that resides symbiotically inside the plant without causing any negative effect to the plant. From the very beginning nature has proved itself a wonderful synthesizer of biologically active molecule. In the past, plants as a whole were highly explored with the expectation that they might harbor some new antibiotics. But from last ten year's endophytic microbes are serving as natural craft man of synthesizing biological moieties, therefore they are gaining momentum in chemical library and in pharmaceutical industries [1,2].

In recent studies it has been found that few proceeding bacterial infection appears to play significant role in chronic disease and leads to fatality. Looking over the world wide incurable diseases and drug resistance bacteria, it's our utmost requirement to continue our search for natural drugs. In order to meet the challenges antibiotic are chemically synthesized either partially (semi synthesis) or completely (total synthesis). But, natural product possesses specific superiority over chemical compounds as they offer unmatched chemical diversity and biological potency over synthetic compounds libraries [3]. Still, $40 \%$ of natural products are not represented in synthetic compound libraries [4]. Therefore various advantageous and novel prospective of pharmacological compounds of endophytic origin over chemically synthesized compounds has captivated researchers toward discovery of microbial metabolites. Hence, isolation and characterization of natural drug from endophytic fungi will always be an innovative and promising requirement of research in field of medical, agriculture and pharmacy. The objective of this work is to isolate and identify the biological active compound extracted from endophytic fungus Emericella qaudrilineata. Different species of Emericella sp. has been a very good source natural product i.e., antimicrobial anti-cancer and anti-diabetic effect. The working strain Emericella qaudrilineata (RS5) contains antibacterial and antifungal activity. Since initial crude extract contains complex mixture of bioactive compounds, therefore isolation is followed by different purification method and spectroscopic technique to characterize the major compound which are beneficial to human and plant pathogens.

\section{Materials and Methods}

Isolation of metabolites from Endophytic strain Emericella qaudrilineata (RS-5): Endophytic fungus coded as RS-5, that had been isolated from medicinal plant Pteris pellucida possesses prominent antibacterial activity against gram positive $S$. aureus (IMS/GN7), gram negative $A$ hydhrophila (IMS/GN11) and antifungal Curvularia sp, Fusarium sp and Cornyespora sp. (Goutam et al.). Sequencing of RS-5 revealed 99\% sequence similarity with Emericella qaudrilineata (Accession number KC662361) [5]. Endophytic fungus was grown for 25 day in 10 different flasks of $1000 \mathrm{ml}$ volume each containing $500 \mathrm{ml}$ of optimized media under static condition. The optimized media for culture (RS-5) was potato dextrose broth supplemented with $1 \%$ starch [5]. After 25 days, harvested culture was filtered via muslin clothes and extracted with optimized organic solvent chloroform [5]. Compounds dissolved in organic solvent were concentrated using rota vapor.

\section{Bioassay guided fractionation of crude metabolite}

It is prerequisite to simplify the range of lead compounds occurrence within the natural complex crude. This method is based on step by step separation of extracted components based on difference

*Corresponding author: Jyoti Goutam, Laboratory of Mycopathology and Microbial Technology, Center of Advance Study in Botany, Banaras Hindu University, Varanasi-221 005, Uttar Pradesh, India, Tel: 945291744; E-mail: Jyoti23biotech@gmail.com

Received February 06, 2016; Accepted February 19, 2016; Published February 26 2016

Citation: Goutam J, Kharwar RN, Tiwari VK, Mishra A, Singh S (2016) Isolation and Identification of Antibacterial Compounds Isolated from Endophytic Fungus Emericella qaudrilineata. Nat Prod Chem Res 4: 205. doi:10.4172/2329 6836.1000205

Copyright: (c) 2016 Goutam J. This is an open-access article distributed under the terms of the Creative Commons Attribution License, which permits unrestricted use, distribution, and reproduction in any medium, provided the original author and source are credited. 
in their physiochemical properties and assuming biological activity followed by next round of separation and assay [6]. In this method whole crude extract is dissolved in methanol in combination with silica ( 3 silica +1 crude extract). Whole mixture is completely dried (sentence deleted). Dried crude extract is fractionated using four solvent of different physiochemical properties (polarity) 100\% hexane, $100 \%$ toluene $100 \%$ ethyl acetate and $70 \%$ methanol. After solvent extraction, antimicrobial activity of each fraction is accessed by specific in vitro disc diffusion assay.

\section{Disc diffusion method}

The fractionated crude extract from E. qaudrilineata (RS-5) was accessed for antibacterial activities by performing disc diffusion method [7]. For this, bacterial suspensions of were made in autoclaved distilled water and their lawns were prepared on Mueller Hinton agar plates with the help of sterile cotton swab. 24 hour old bacterial culture of gram positive Staphylococcus aureus and gram negative Aeromonas hydrophila were used for making bacterial suspension. Sterile disc impregnated with fractionated extract of different solvent at the concentration of $1 \mathrm{mg} /$ disc were placed on the lawn culture and observed for the zone of inhibition after 48 hours. The zone of inhibition against reported bacterial pathogens was measured by scale.

\section{Purification and separation of compound via column chromatography}

Purification and separation of compounds were performed using methodology of Devi. Column chromatography (CC) was undertaken in a glass column $(700 \mathrm{~mm} \times 30 \mathrm{~mm})$. Silica gel $(100-120$ mess size Merk) was used as stationary phase. Movable phase consisted of pure solvent or different solvents depending upon requirement of conditions. Column was loaded with crude complex extracted from endophytic fungus (RS-5). Mobile phase consist of toluene and hexane in 5: 95, 10: 90, 20: 80, and 30: 70 ratio was used for separating compound and gradient elution was followed. Different fractions eluted from column chromatography (CC) were separated by thin layer chromatography (TLC) and checked for antibacterial activity.

\section{Analytical Thin Layer Chromatography (TLC)}

Thin layer chromatography (TLC) was performed on $60 \mathrm{~F}_{25}$ silica gel, pre-coated on aluminum plates(Merk) and was revealed with either a UV lamp $\left(\lambda_{\max }=254 \mathrm{~nm}\right)$ or a specific colour reagent (Dragendorff reagent or iodine vapors) or by spraying with methanolic$\mathrm{H}_{2} \mathrm{SO}_{4}$ solution and subsequent charring by heating at $100^{\circ} \mathrm{C} .100 \mathrm{ml}$ fractions of solvent fractions were collected while performing column chromatography. All solvents were of pure analytical grade. Eluted solvents were evaporated under reduced pressure on IKA Rotary Evaporator at temperature $<50^{\circ} \mathrm{C}$. From each fraction $10 / \mu \mathrm{g} / \mu \mathrm{l}$ of compound was spotted using fine capillary tubes, and the spots were detected in UV Chamber. Mobile phase used in TLC were consist of 20 toulene: 80 hexane.

\section{Elucidation of structure via spectroscopic analysis}

It is difficult to access the original compound of interest present in natural products which is usually a complex mixture of several compounds. Hence two important techniques were undertaken to reach the structure of antimicrobial compounds i.e., GCMS and NMR spectroscopy. GC-MS analysis was carried out in GCMS QP 2010 plus Shimadju gas chromatograph coupled with a series mass selective detector and pressure was kept $82.3 \mathrm{kPa}$. The column temperature was $70^{\circ} \mathrm{C}$ initially with a hold of $4 \mathrm{~min}$, then programmed to $250^{\circ} \mathrm{C}$ at a rate of $5^{\circ} \mathrm{C} / \mathrm{min}$ for $5 \mathrm{~min}$ and programmed to $320^{\circ} \mathrm{C}$ for $12 \mathrm{~min}$ at the rate of $20 \mathrm{~min} / \mathrm{sec}$. Helium was used as the carrier gas and the column head pressure was maintained at $13.3 \mathrm{psi}$. Injector temperature was at maintained $260^{\circ} \mathrm{C}$, and the injection volume was $1.21 \mathrm{ml} / \mathrm{min}$ in the split mode. The interface temperature was held at $280^{\circ} \mathrm{C}$. Mass spectra were scanned from m/z 40 to 600 with a scan speed of 1250 . Data were analysed using GCMS solution software along with concerning the library of WILEY and NIST.

\section{Nuclear Magnetic Resonance spectroscopy (NMR)}

Extensive spectroscopic data $\left({ }^{1} \mathrm{H}\right.$ and ${ }^{13} \mathrm{C}$ NMR $)$ were recorded on JEOL AL300 FT-NMR Spectrometer at 300 and $75 \mathrm{MHz}$, respectively. Chemical shifts given in ppm downfield from tetramethylsilane TMS $(\delta=0.0 \mathrm{ppm})$ or solvent as internal reference; J values in $\mathrm{Hz}$. Solvents were $\mathrm{D}_{2} \mathrm{O}$ and DMSO- $\mathrm{d}_{6}$.

\section{Fourier Transform Infrared Resonance spectroscopy (FTIR)}

Infrared spectra recorded as Nujol mulls in $\mathrm{KBr}$ plates. IR spectral data was recorded on FTIR-8201 PC Shimadzu spectrometer.

\section{Statistical analysis}

Antibacterial assessment during bioassay guided fractionation was done in triplicate. Value of data was expressed as mean \pm S.D. from triplicate value. $\mathrm{P}$ values of 0.05 or less were considered statistically significant.

\section{Results and Discussion}

Fungal endophytes have been recognized as prolific producer of many chemical compounds having antibacterial, antifungal other biological potential $[8,9]$. Many endophytic fungi have been exploited for their active potential. Among them different species of Emericella sp. has been a very good source innumerable potential natural compound. It is well known crude extract isolated from these endophytic fungal metabolites contains complex chemical diversity which is difficult to identify and characterize. That's why purification and characterization of potential compound needs a very systematic approach. In this study isolation and identification strategy of bioactive crude complex lead to isolation of benzyl benzoate from the Emericiella quadrilineata derived from fern Pteris pellucida from Arunachal Pradesh India. In past, this compound has been reported from different plant extract containing various physiological activities such as spasmolytic [10], calmodulin inhibitor [11], tyrosinase inhibitors [12]. First time it has been isolated from endophytic fungus Emericiella quadrilineata. It was first used to treat Angina pectoris [13]; Benzyl benzoate is one of the primary prescribed ointments to treat scabies $[14,15]$.

Bioassay guided fractionation: Endophytic fungus Emericiella quadrilineata responsible for antibacterial and antifungal activity against $\mathrm{Sa}, \mathrm{Ah}$, Fusarium oxysporum, Curvularia sp, Altermaria alternata was isolated and identified [5]. Since endophytes keep high arrangement of bioactive molecules complexity in their secondary metabolites therefore it is required to pick out relevant information from the large amount of data from known physiological assay (bioassay guided fractionation [16]. Usually bioassay guided fractionation were performed in plant extracts as their secondary metabolites contains diverse chemical compounds. Benzyl benzoate and its various derivatives have isolated from extract of dichloromethane from Uvaria pauci-ovulata bark [17]. Collected crude extract was fractionated into different fractions using different organic solvents range from lower to higher polarity. On accessing biological activity, toluene solvent was found to contain major compound of antibacterial interest followed by ethyl acetate and 
hexane as it showed maximum antibacterial activity with $22 \pm 0.8 \mathrm{~mm}$ and $15 \pm 0.8 \mathrm{~mm}$ against gram positive Staphylococcus aureus and gram negative Aeromonas hydrophila respectively. Disk loaded with only hexane, toluene ethyl acetate and methanol as negative control (Figure 1; Tables 1 and 2). No activity exhibited from methanol extract, hence it can be concluded that fraction that was only soluble in methanol was not playing any role in inhibiting pathogenic bacteria $S$. aureus and $A$ hydrophila.

\section{Gas chromatography mass spectroscopy (GCMS)}

In next part of characterization, active fraction (from toluene) evaluated by GCMS (gas chromatography mass spectroscopy) technique found Benzyl benzoate (27.3\%), Benzaldehyde dimethyl acetal (15.04\%) and Benzoic acid (17\%) as major compound (Figures 2 and 3$)$.

\section{Column chromatography (CC), Thin Layer Chromatography (TLC) and Disc diffusion assay}

Most active fraction resulted from toluene were subjected to column chromatography technique. Total 25 fractions eluted in the ratio of 20 Toluene: 80 Hexane from column. Each fraction dried and resolved as a single compound at $0.5 \mathrm{RF}$ value in TLC under UV lamp (Figure 4a). TLC separated compound exhibited $18 \mathrm{~mm}$ zone of inhibition against $S a$ as compared to $25 \mathrm{~mm}$ in toluene crude (Figure 4c) and $15 \mathrm{~mm}$ zone of inhibition against $A h$ as compared to $20 \mathrm{~mm}$ in toluene crude (Figure $4 \mathrm{~b}$ ). It can be concluded that there was little decrease in activity with respect to major active crude of toluene against gram negative A. hydrophila and gram positive S. aureus (Figure $4 \mathrm{~b}$ and 4c). The concentration of compound loaded in above disk was $1 \mathrm{mg} / 10$ $\mu$ l. Hence separated compound was found to containing major activity against bacterial pathogens.

\section{Nuclear magnetic resonance spectroscopy (NMR) and Infrared spectroscopy (IR)}

Single compound detected in TLC was collected and analyzed by one dimension ${ }^{1}$ proton and ${ }^{2}$ carbon NMR and supported the GCMS data of toluene fraction. NMR spectra of compound separated in TLC from column indicated that it could probably be a mixture of benzyl benzoate and benzaldehyde as per spectroscopic data predictions; A singlet at $\delta$ 9.93 integrated to one proton was identified for an aldehydic proton. A multiplet appearing in the range of $\delta 7.87-7.20$ indicated the presence of 15 aromatic protons. A sharp singlet integrated to two protons at $\delta 5.01$ evidenced the presence of $O$-methylene group. Additionally,

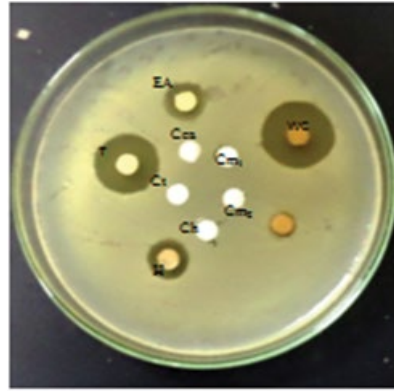

A

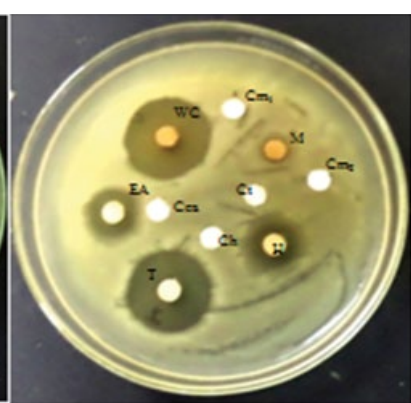

$\mathrm{B}$
Figure 1: Antibacterial assay via disc diffusion method (Aeromonas hydrophilla (A) Staphylococcus aureus (B) (T-Toluene), (EA-Ethyl acetate), (W-whole crude extract), (M-Methanol), (Hexane-H), (Cea-Control of ethyl acetate), $\left(\mathrm{Cm}_{1}\right.$ Control of methanol at $50 \%)$, (Ch-Control of hexane), (Ct-Control of Toluene), $\left(\mathrm{Cm}_{2}\right.$-Control of methanol at $\left.\left.50 \%\right)\right)$.

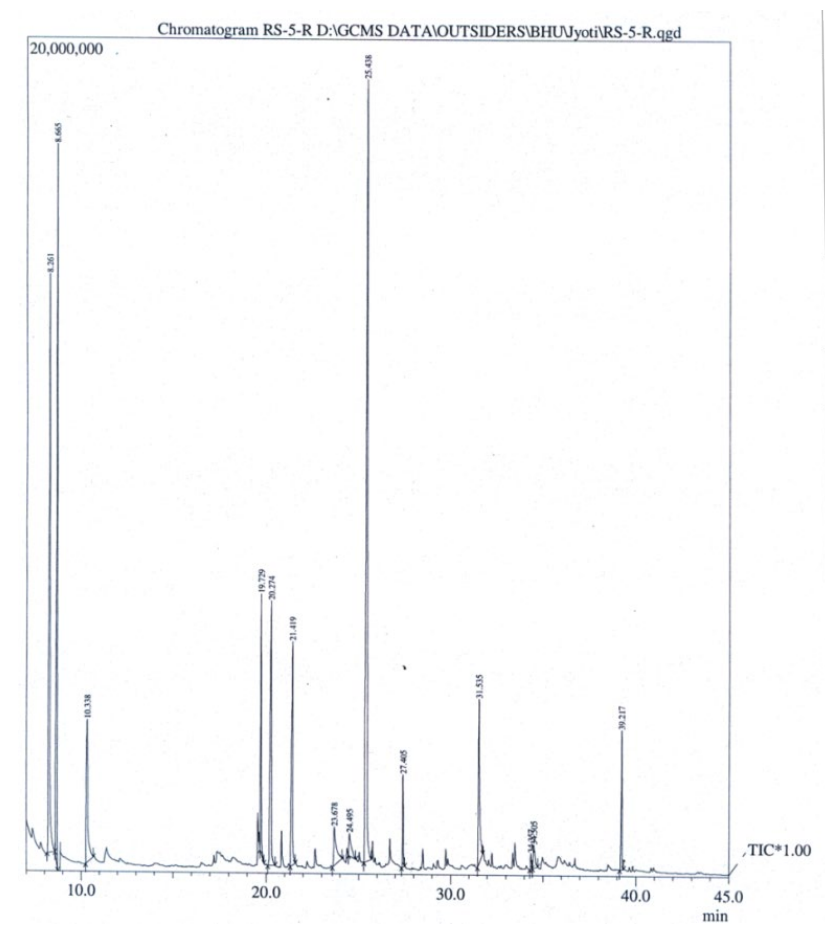

Figure 2: GCMS profile of Toluene fraction<smiles>O=C(OCc1ccccc1)c1ccccc1</smiles>

Benzyl benzoate

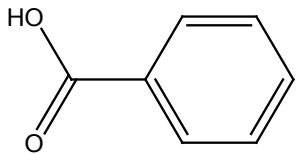

Benzoic acid

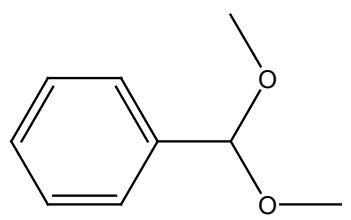

Benzaldehyde dimethyl acetal

Figure 3: Structure of major compounds expected from toluene fraction.

\begin{tabular}{|l|l|l|l|}
\hline S No & Solvent & $\begin{array}{l}\text { ZOI Against Sa }(\mathbf{m m}) \\
\text { Mean } \pm \text { SD }\end{array}$ & $\begin{array}{l}\text { ZOI Against Ah }(\mathbf{m m}) \\
\text { Mean } \pm \text { SD }\end{array}$ \\
\hline 1 & Hexane $(\mathrm{H})$ & $14.5 \pm 0.7$ & $8.5 \pm 1.2$ \\
\hline 2 & Toluene $(\mathrm{T})$ & $22 \pm 0.8$ & $15 \pm 0.8$ \\
\hline 3 & $\begin{array}{l}\text { Ethyl acetate } \\
(\mathrm{EA})\end{array}$ & $10 \pm 0.8$ & $9 \pm 0.8$ \\
\hline 4 & Methanol (M) & Not active & Not active \\
\hline 5 & $\begin{array}{l}\text { Crude extract } \\
\text { (WE) }\end{array}$ & $24.6 \pm 1.2$ & $17 \pm 1.6$ \\
\hline
\end{tabular}

Table 1: Bioactivity guided assay of each fraction against targeted bacterial pathogens.

the ${ }^{13} \mathrm{C}$ NMR showed the presence of many peaks among which the aromatic carbons appeared at $\delta$ 135.8-125.5. The aldehydic carbons appeared at $\delta 192.5$ and the $O$-methylene carbon appeared at $\delta 64.5$. In the IR spectra further the presence of carbonyl group was confirmed by the appearance of a sharp absorption band at $1728 \mathrm{~cm}^{-1}$. (Figure 
Citation: Goutam J, Kharwar RN, Tiwari VK, Mishra A, Singh S (2016) Isolation and Identification of Antibacterial Compounds Isolated from Endophytic Fungus Emericella qaudrilineata. Nat Prod Chem Res 4: 205. doi:10.4172/2329-6836.1000205

Page 4 of 7

\begin{tabular}{|l|l|l|l|}
\hline Peak & RT & Area $(\mathbf{\%})$ & Name of compounds \\
\hline 1 & 8.261 & 17.41 & Benzoic acid, methyl ester \\
\hline 2 & 8.665 & 15.04 & Benzaldehyde dimethyl acetal \\
\hline 3 & 10.338 & 5.65 & Dehydromevalonic lactone \\
\hline 4 & 19.729 & 4.98 & BENZENE, 1,1'-(1,2-ETHANEDIYL)BIS- \\
\hline 5 & 20.274 & 8.38 & Cyclohexanol, 1,3-dimethyl-, cis- \\
\hline 6 & 21.419 & 6.88 & ACETIC ACID 2-(3-METHYL-OXIRANYL)-6-OXO-3 \\
\hline 7 & 23.678 & 2.42 & Heptanoic acid \\
\hline 8 & 25.438 & 27.32 & Benzyl benzoate \\
\hline 9 & 27.405 & 1.66 & 1,2 Benzenedic dicarboxylic acid \\
\hline 10 & 31.535 & 5.03 & 2,2 Oxydibenzaldehyde \\
\hline 11 & 34.505 & 1.08 & 4-(4-Methylaminobenzylideneamino) benzonitrile \\
\hline 12 & 39.217 & 2.59 & 1,2 Benzenedic dicarboxylic acid \\
\hline
\end{tabular}

Table 2: Major Compounds enlisted from Toluene fraction via GCMS analysis.

\section{Separation of compound in TLC and its antibacterial assay against targeted bacterial pathogens}
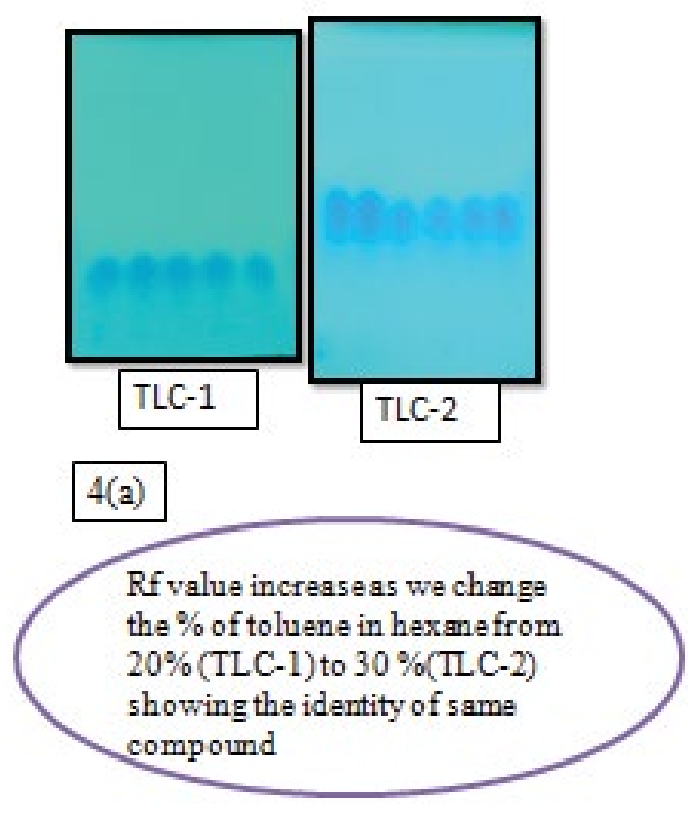
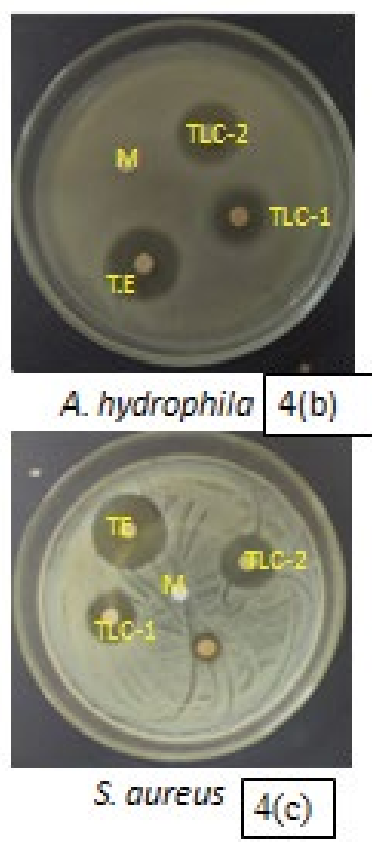

Figure 4: Separation of compound in TLC plates and its antibacterial assay with respect to toluene extract (TE).

$5 a, 5 b$ and $5 c$ ) Mass spectrum of compound 1 has been represented in Figure 6. A number of compounds were recorded according to GCMS prediction, but major compound indicated by above evidences in most active fraction was recognized and identified as Benzyl benzoate. Trade name of Benzyl benzoate is Ascabiol, used as scabicides and other body lotion. Benzyl benzoates and its derivatives (benzyl benzoate, benzyl cinnamate and meta-methyl and 3-methylbenzyl 2-nitrobenzoate) also reduce hypertension [18]. Based on described work a number of chemical characterizations of bioactive compounds have been reported from different endophytic fungi as Penicillium chrysogenum [19], volatile organic compound from Muscodar Albus [20], and javanicin napthaquinone from choridium [21].
In context to our study it could be concluded that Benzyl benzoates and its various analogues derivatives of this compound have been isolated from Emericiella qaudrilineata (RS-5) has also been reported from plant metabolites and its effect on human being [18]. Hence with respect to our result still chemists are required to form synthetic derivative of compound (combinatorial chemistry) in order to explore other bioactive principles and their applications on human being [22]. Combinatorial chemistry emerged as a "white knight" with the potential to address major issues of natural product and lead molecules in pharmaceutical industries [23].

\section{Conclusion}

Endophytes use food material from plant and synthesize diverse 


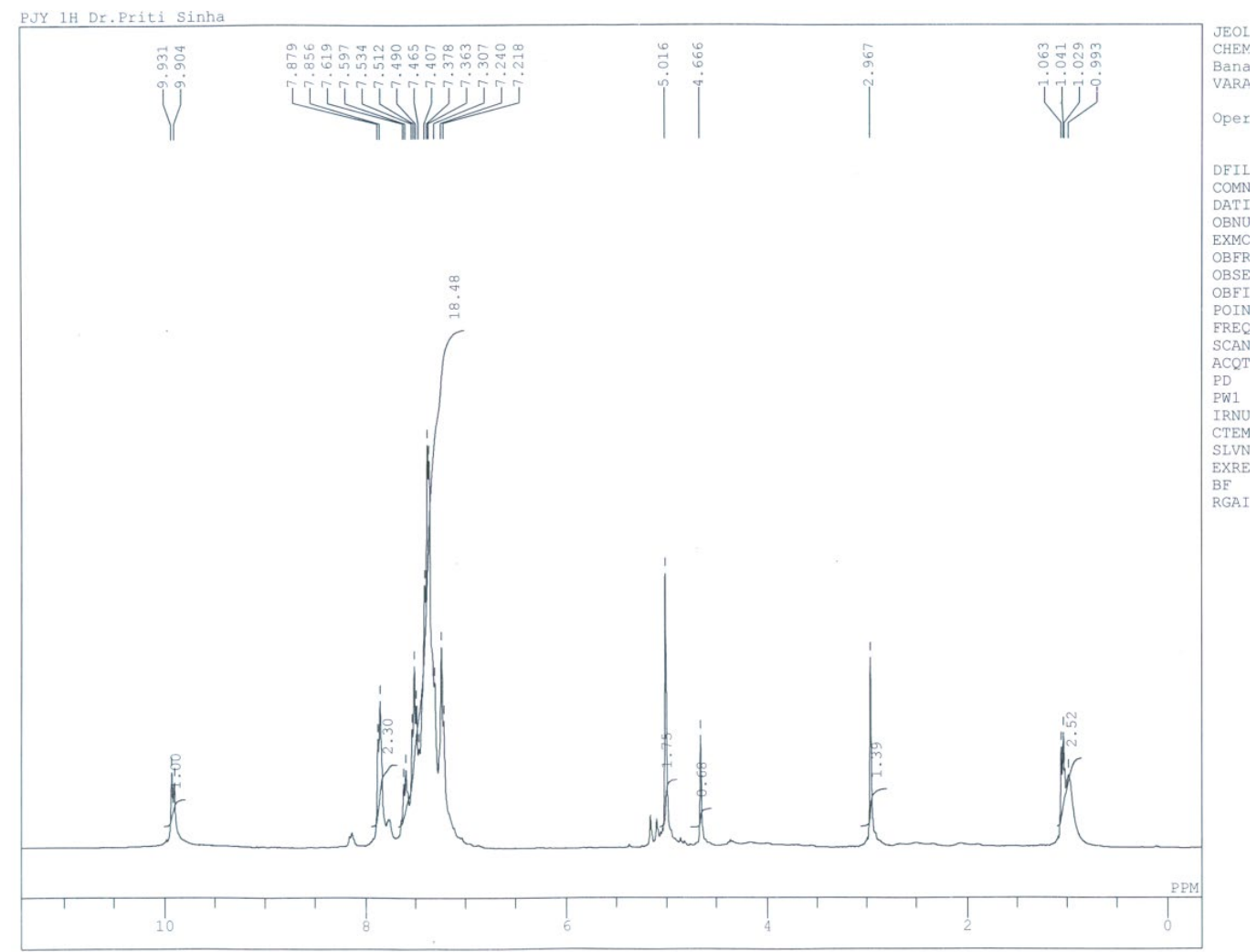

Figure 5a: Proton NMR of compound observed in TLC Figure 4.

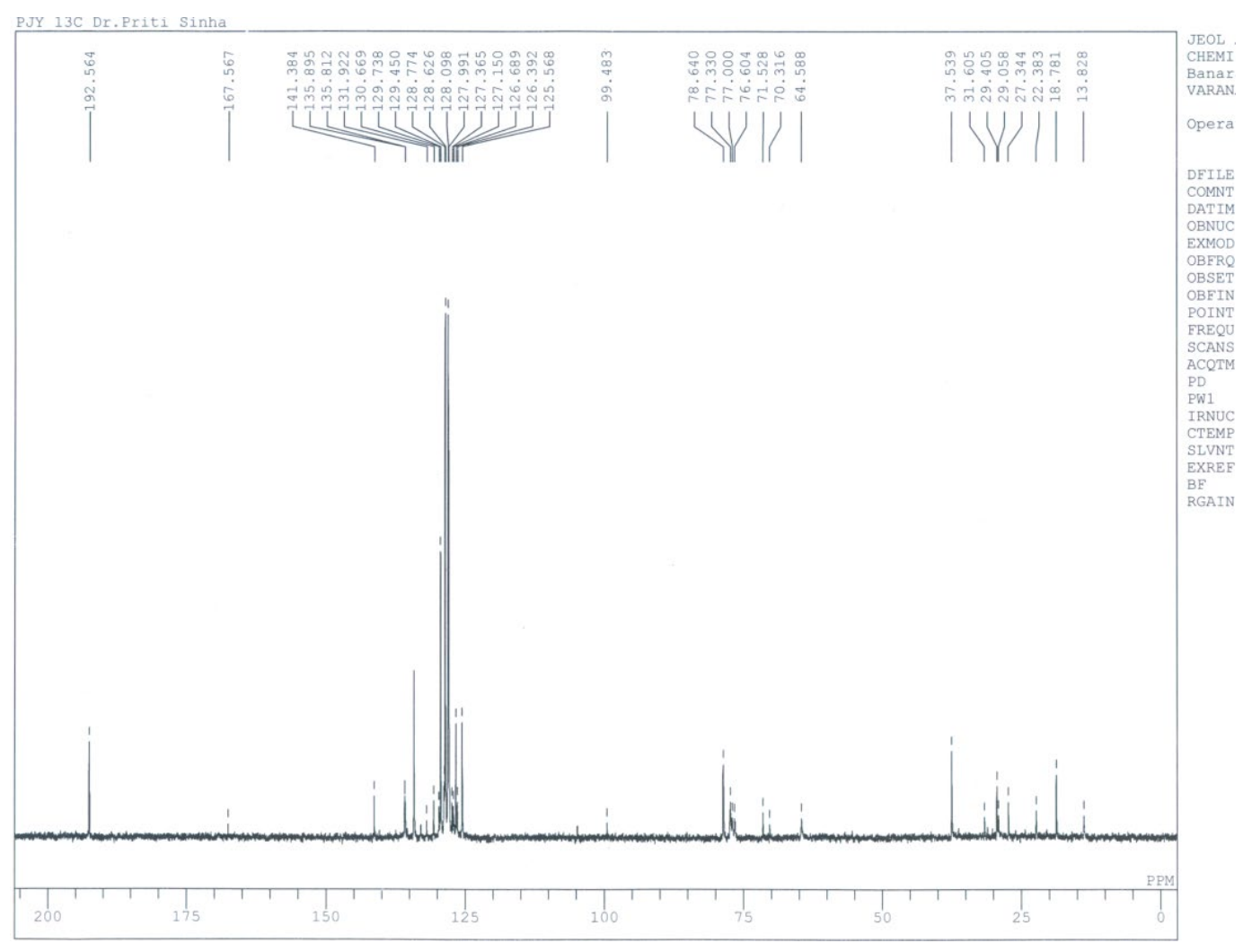

Figure 5b: $\mathrm{C}^{13}$ NMR of compound observed in TLC Figure 4. 
Citation: Goutam J, Kharwar RN, Tiwari VK, Mishra A, Singh S (2016) Isolation and Identification of Antibacterial Compounds Isolated from Endophytic Fungus Emericella qaudrilineata. Nat Prod Chem Res 4: 205. doi:10.4172/2329-6836.1000205

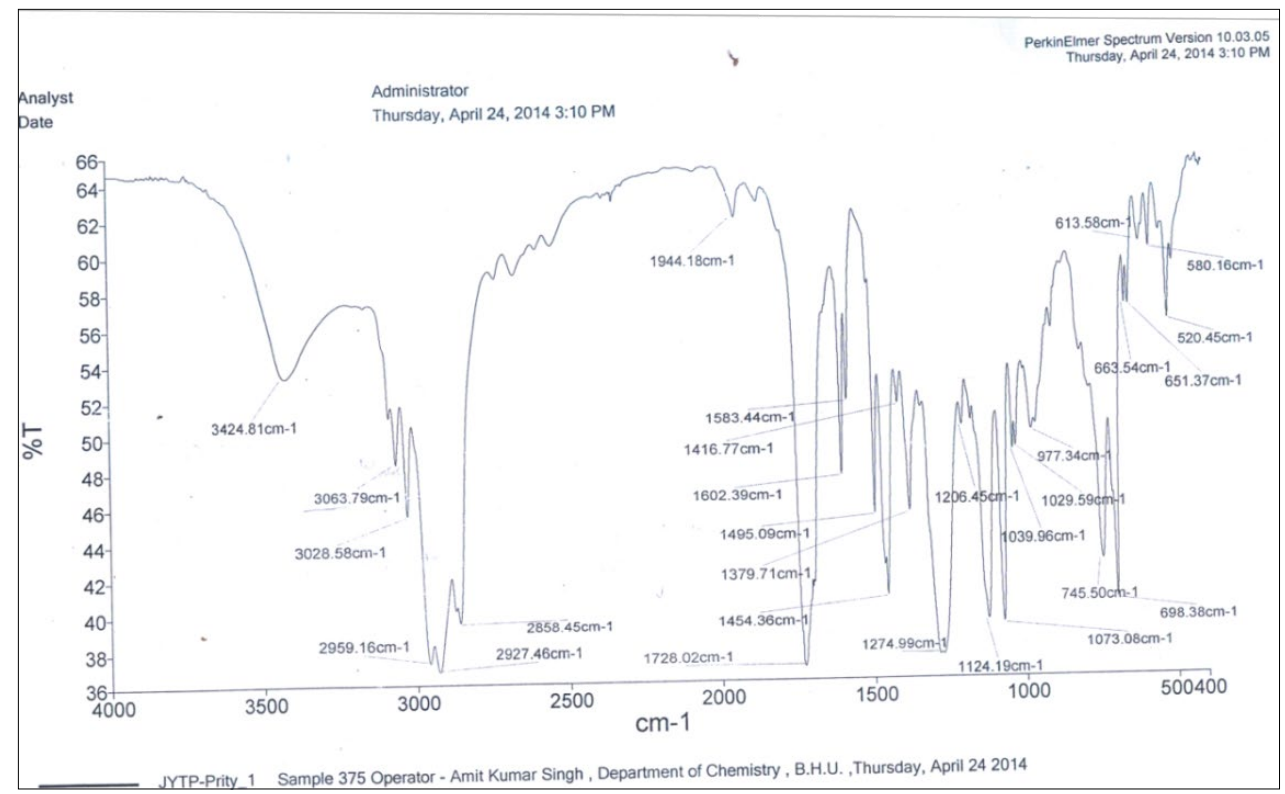

Figure 5c: FTIR analysis of compound analyzed in Figure 4 in TLC plates.

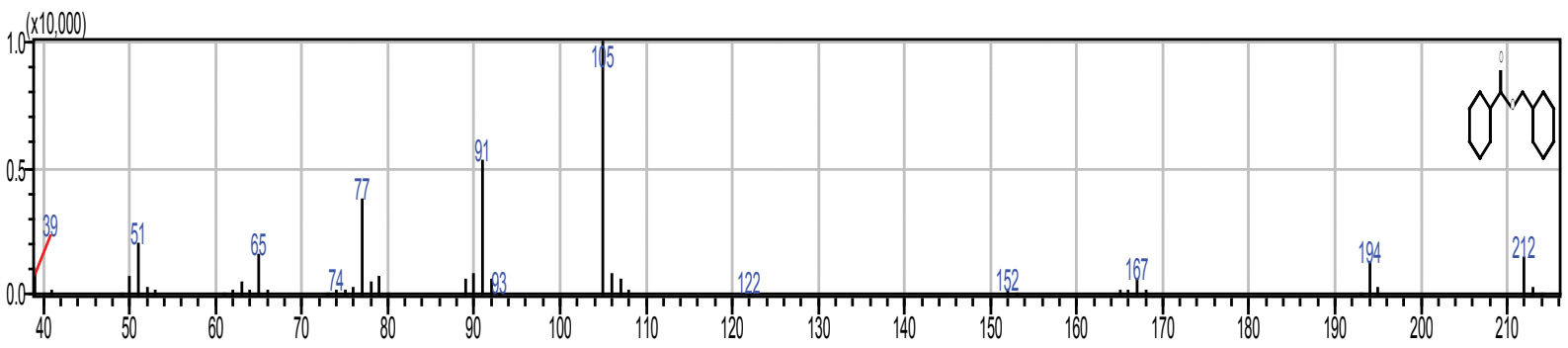

Figure 6: Mass chromatogram of compound 1Benzyl benzoate (212 Dalton).

array of molecules from simple to complex with their different physiological mode of action. Endophyte produces metabolites insides the plant which can be used for mankind after exploiting in in-vitro conditions. In respect of above context it could be said the might be fungal and plant interaction for food and defense mechanism respectively. Hence utilizing proper methodology we can access to major compound of interest.

\section{Acknowledgments}

The authors are thankful to the following: The Head and Coordinator, CAS in Botany, BHU, Varanasi, for facilities; the Council of scientific industrial research (CSIR) and Union grant commission (UGC), New Delhi, for financial support as JRF and SRF; the Department of science and technology (DST), New Delhi, for financial support to RNK as project (File No. SR/SO/PS-78/2009, date: 10-5-2010. Authors thank to Prof. Gopal Nath (IMS, BHU) and Prof. R. Chand (IAS, BHU) Varanasi for help in antibacterial and antifungal assays. Authors are also thankful to Ajay Kumar for GCMS JNU AIRF Facility and CIL Department of chemistry BHU.

\section{References}

1. Zhang HW, Song YC, Tan RX (2006) Biology and chemistry of endophytes. Nat Prod Rep 23: 753-771.

2. Kaul S, Gupta S, Ahmed M (2012) Endophytic fungi from medicinal plants: a treasure hunt for bioactive metabolites. Phytochem Rev 11: 487-505.

3. Zhang L, Demain AL (2005) Natural Products: Integrated approach for discovering novel drugs from microbial natural products. Natural product: Drug
Discovery and Therapeutic Medicine. Inc., Totowa, NJ, Springer Humana Press.

4. Satish S, Baker S (2012) Endophytes: Natural warehouse of bioactive compounds. Drug invention today 4: 548-553.

5. Goutam J, Sharma VK, Verma SK, Singh DK, Kumar J, et al. (2014) Optimization of cultural condition for enhanced production of bioactive metabolites rich in antimicrobial and antioxidant Activities isolated from Emericella quadrilineata an endophyte of Pteris pellucida. J Pure App Microbiol 8: 2059-8073.

6. Yang X, Summerhurst DK, Koval SF, Ficker C, Smith ML, et al. (2001) Isolation of an antimicrobial compound from Impatiens balsamina L. using bioassayguided fractionation. Phytother Res 15: 676-680.

7. Bauer AW, Kirby WM, Sherris JC, Turck M (1966) Antibiotic susceptibility testing by a standardized single disk method. Am J Clin Pathol 45: 493-496.

8. Fisher PJ, Anson AE, Petrini O (1989) Antibiotic activity of some endophytic fungi from ericaceous plants. Botanica Helvetica 93: 508-512.

9. Hoffman AM, Mayer SG, Strobel GA, Hess WM, Sovocool GW, et al. (2008) Purification, identification and activity of phomodione, a furandione from an endophytic Phoma species. Phytochemistry 69: 1049-1056.

10. Rivero-Cruz B, Rivero-Cruz I, Rodríguez-Sotres R, Mata R (2007) Effect of natural and synthetic benzyl benzoates on calmodulin. Phytochemistry 68 1147-1155.

11. Figueroa M, González Mdel C, Rodríguez-Sotres R, Sosa-Peinado A González-Andrade M, et al. (2009) Calmodulin inhibitors from the fungus Emericella sp. Bioorg Med Chem 17: 2167-2174. 
Citation: Goutam J, Kharwar RN, Tiwari VK, Mishra A, Singh S (2016) Isolation and Identification of Antibacterial Compounds Isolated from Endophytic Fungus Emericella qaudrilineata. Nat Prod Chem Res 4: 205. doi:10.4172/2329-6836.1000205

Page 7 of 7

12. Fang $Y$, Chen $Y$, Feng G, Ma L (2011) Benzyl benzoates: New phlorizin analogs as mushroom tyrosinase inhibitors. Bioorg Med Chem 19: 1167-1171.

13. Robert H, Babcock MD (1924) The use of Benzyl- benzoate in the treatment of Angina Pectoris. JAMA 82: 193-196.

14. Gilman AG, Goodman LS, Gilman A (1980) Benzyl benzoate usp is used as $25 \%$ lotion Goodman and Gilman's, The Pharmacological Basis of Therapeutics Macmillan, New York, USA.

15. Bachewar NP, Thawani VR, Mali SN, Gharpure KJ, Shingade VP, et al. (2009) Comparison of safety, efficacy, and cost effectiveness of benzyl benzoate, permethrin, and ivermectin in patients of scabies. Indian J Pharmacol 41: 9-14.

16. Weller MG (2012) A unifying review of bioassay-guided fractionation, effectdirected analysis and related techniques. Sensors (Basel) 12: 9181-9209.

17. Raynaud S, Fourneau C, Laurens A, Hocquemiller R, Loiseau P, et al. (2000) Squamocin and benzyl benzoate, acaricidal components of Uvaria pauciovulata bark extracts. Planta Med 66: 173-175
18. Ohno O, Ye M, Koyama T, Yazawa K, Mura E, et al (2008) Inhibitory effects of benzyl benzoate and its derivatives on angiotensin II-induced hypertension. Bioorg Med Chem 16: 7843-7852.

19. Devi $P$, Rodrigues C, Naik CG, D'Souza L (2012) Isolation and Characterization of Antibacterial Compound from a Mangrove-Endophytic Fungus, Penicillium chrysogenum MTCC 5108. Indian J Microbiol 52: 617-623.

20. Ezra D, Hess WM, Strobel GA (2004) New endophytic isolates of Muscodor albus, a volatile-antibiotic-producing fungus. Microbiology 150: 4023-4031

21. Kharwar RN, Verma VC, Kumar A, Gond SK, Harper JK, et al. (2009) Javanicin, an antibacterial naphthaquinone from an endophytic fungus of neem, Chloridium sp. Curr Microbiol 58: 233-238.

22. Bleicher KH, Böhm HJ, Müller K, Alanine Al (2003) Hit and lead generation: beyond high-throughput screening. Nat Rev Drug Discov 2: 369-378.

23. Kennedy JP, Williams L, Bridges TM, Daniels RN, Weaver D, et al. (2008) Application of combinatorial chemistry science on modern drug discovery. $\mathrm{J}$ Comb Chem 10: 345-354. 